\title{
1D-3D Registration for Functional Nuclear Imaging
}

\author{
Christoph Vetter ${ }^{1,2}$, Tobias Lasser ${ }^{1}$, Thomas Wendler ${ }^{1,3}$, and Nassir Navab ${ }^{1}$ \\ 1 Chair for Computer Aided Medical Procedures (CAMP), \\ Technische Universität München, Germany \\ 2 Siemens Corporate Research, Princeton, USA \\ 3 SurgicEye GmbH, Munich, Germany
}

\begin{abstract}
Functional nuclear imaging systems like PET or SPECT provide unique information that is used extensively in diagnosis, but it has also proven very useful for image-guided interventions. In the case of SPECT and radio-guided surgeries, 1D gamma detectors called gamma probes are routinely used during interventions to localize hotspots in conjunction with pre-operative SPECT images, or more recently, intraoperative SPECT images. As the tissue is being manipulated during surgery, these SPECT images quickly lose their validity, necessitating either new scans, which is in most cases unfeasible, or requiring the surgeon to do a mental update of the available imagery. In this paper, we present a novel 1D-3D registration procedure for functional nuclear imaging that registers tracked intra-operative $1 \mathrm{D}$ probe readings to a pre- or intra-operatively acquired 3D functional image. This procedure allows incorporating prior knowledge during radio-guided surgeries, enabling rapid updates of the visualization in the case of tissue deformation without the overhead of an additional complete scan. We show results using phantom data as well as patient data.
\end{abstract}

\section{Introduction}

Functional nuclear imaging modalities like PET or SPECT provide specific information to localize in 3D even very small tumors in several oncological applications [23]. Translating this information to the operating room however faces several difficulties: the patient posture may change, treatment in between the imaging and surgery might affect the region of interest and finally, tissue manipulation during surgery invalidates the previous imaging information [1].

In the case of SPECT and radio-guided surgeries, 1D gamma detectors (also called gamma probes) are routinely used during interventions to localize hotspots in conjunction with pre-operative SPECT images, or more recently, intraoperative SPECT images [7] (see figure 11). The count rates from the gamma probe, presented visually as well as acoustically (like a Geiger counter), help the surgeon translating in his mind the available functional imaging information to the current situation.

G. Fichtinger, A. Martel, and T. Peters (Eds.): MICCAI 2011, Part I, LNCS 6891, pp. 227-234, 2011.

(C) Springer-Verlag Berlin Heidelberg 2011 
In this work we suggest a novel, automatic $1 \mathrm{D}-3 \mathrm{D}$ registration procedure to register 1D gamma probe readings, augmented by synchronized position and orientation data acquired by an optical tracking system, to pre- or intra-operative 3D SPECT imaging data, which has been invalidated due to tissue manipulation during surgery. We demonstrate our approach for the use case of sentinel lymph node biopsies (SLNB) in breast cancer. Sentinel lymph node biopsy is a technique to determine if breast cancer has spread into the lymphatic system in a low morbidity way and is now standard practice for early stage breast cancer patients [5]. In the SLNB procedure a low-energy radioactive tracer for SPECT is injected near the tumor, and the lymph nodes showing radioactivity as for example indicated by a hand-held gamma probe (i.e. the first nodes in the lymph drainage path of the tumor) are then excised and examined histologically for cancerous cells. Given the presence or absence of metastases in the excised nodes, different treatments are indicated making this technique of major importance in cancer staging. The possibility of selectively excising the first nodes in the drainage of the tumor with the help of this nuclear medicine approach allows better targeted surgery, less morbidity and shorter hospital stays for the patient. Technical advancements like 3D intra-operative SPECT imaging are poised to improve the accuracy of the SLNB procedure [6], but even there tissue manipulation during surgery poses difficulties that can be addressed using the introduced 1D-3D registration procedure in this work.

The technique suggested here is one step towards a future in which surgical instrument containing integrated detectors provide a constantly updated view of the radioactivity in tissue, allowing real-time updates of the guidance for the surgeon, which results in faster and more efficient surgery as well as a better quality control. This paper presents the methods employed for the proposed 1D$3 \mathrm{D}$ registration procedure and evaluates the procedure using a series of phantom experiments as well as patient data.

\section{Methods}

\subsection{Input Data and Output}

The registration method requires two inputs, a three-dimensional volume $V \subset$ $\mathbb{R}^{3}$ (for example SPECT) and a series of tracked 1D probe measurements $b_{j} \in \mathbb{R}$, $j=1, \ldots, k$ (for example from a gamma probe), which are acquired during the surgery. Here $b_{j}$ denotes the count rate (counts per second) of the probe, and associated with each measurement $b_{j}$ are the synchronized position and orientation $\left(p_{j}, o_{j}\right) \in \mathbb{R}^{3} \times \mathbb{R}^{3}$ as acquired by the tracking device. The output of the method will be an updated $3 \mathrm{D}$ volume $\widehat{T}(V)$, where $\widehat{T}: \mathbb{R}^{3} \rightarrow \mathbb{R}^{3}$ is an approximation of the optimal transformation transforming the no longer valid volume $V$ into the desired volume $\widehat{T}(V)$.

The challenge in this registration method lies in the very limited number of probe measurements covering only a fraction of the volume of interest due to time and practical constraints. Several other factors also add complications, 

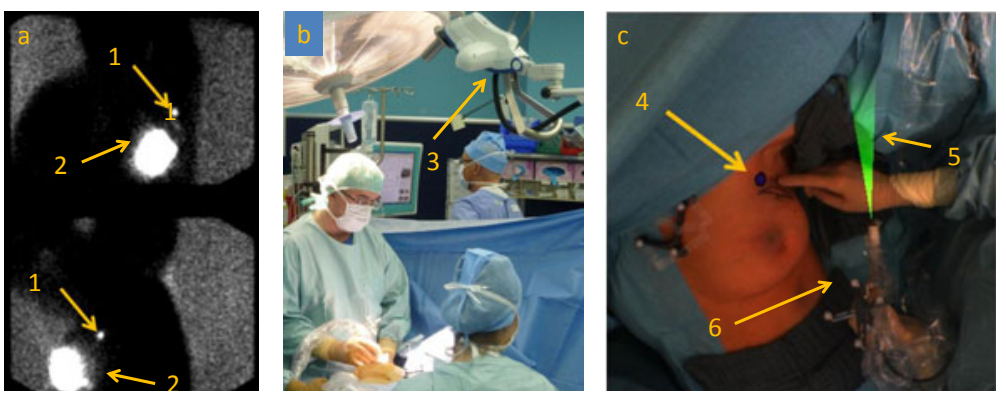

Fig. 1. a) shows two pre-operative projection angles of a scintigraphy for SLNB (top: frontal, bottom: left lateral) with the lymph node (1), and the injection site (2), b) shows the workstation with the gamma probe and the tracking device (3) during a SLNB procedure, c) shows an augmented reality view of the surgery area. The visual guidance overlay consists of the node in blue indicating the lymph node (4) and the triangle in green, indicating the field of view of the probe (5). The hand-held gamma probe is marked as 6 .

namely the statistical nature of the detection process, the large field of view of the gamma probe as well as inaccuracies of the tracking system along with the lack of information of the tissue's attenuation and scattering properties.

In the particular case of SLNB, the probe measurements are typically acquired using gyrating movements around the suspected location of a lymph node, starting at the old location indicated by the SPECT, moving closer to the current location as guided by the probe measurements via visual or acoustic feedback. This means that large parts of the SPECT volume are not covered by probe measurements and that many measurement angles are missing.

To cope with this, the registration method partitions the SPECT volume into a fixed part $V_{f}$ and a moving part $V_{m}, V_{f} \cup V_{m}=V, V_{f} \cap V_{m}=\emptyset$. This is accomplished by segmenting the lymph nodes $\mathcal{N}=\left\{N_{1}, \ldots, N_{l}\right\}$ in the SPECT volume, choosing the lymph nodes $\mathcal{M} \subset \mathcal{N}$ which are closest to the probe measurement positions $p_{j}$ and then forming $V_{m}=\bigcup_{M \in \mathcal{M}} \operatorname{bbox}(M)$ as the union of spherical bounding boxes around the nodes in $\mathcal{M}$. We set $V_{f}=V \backslash V_{m}$. The actual registration procedure is then only applied to $V_{m}$, that is, the output of the method is $\widehat{T}\left(V_{m}\right)$, where $\widehat{T}: V_{m} \rightarrow \mathbb{R}^{3}$ reuses the previous notation of the unrestricted $\widehat{T}$.

\subsection{Registration Procedure}

For choosing a deformation model, it is important to note that the deformation model can only incorporate information that is provided by the nuclear probe. Even though the unlabeled tissue around the radioactively labeled nodes restricts the nodes' movement, no information about this tissue is available. In particular, surgical operations like cutting or cauterizing change tissue and tissue connectivity. Hence our deformation model treats each node in $\mathcal{M}$ as moving 
independently. Furthermore, each node in $\mathcal{M}$ is assumed to not deform from its spherical shape. The deformation model then ends up as pure translations $\left.\widehat{T}\right|_{M}$ for each $M \in \mathcal{M}$.

For the actual registration, the probe measurements $b_{j}$ act as the fixed image, while the moving image are measurements simulated from the current transformed volume $T\left(V_{m}\right)$, where $T: V_{m} \rightarrow \mathbb{R}^{3}$ denotes the current estimate of $\widehat{T}$. The similarity measure employed is sum of squared differences (SSD). This results in the minimization of

$$
\widehat{T}=\operatorname{argmin}_{T} \sum_{j=1}^{k}\left(P_{j}\left(T\left(V_{m}\right)\right)-b_{j}\right)^{2}+\operatorname{reg}\left(T\left(V_{m}\right)\right),
$$

where $P_{j}\left(T\left(V_{m}\right)\right)$ denotes the $j$-th simulated measurement from $T\left(V_{m}\right)$, and $\operatorname{reg}\left(T\left(V_{m}\right)\right)$ denotes a regularization term. In this work we used $\operatorname{reg}\left(T\left(V_{m}\right)\right)=$ $\left\|T\left(V_{m}\right)\right\|_{2}^{2}$.

The resulting minimization problem can be solved with standard optimization methods. For this work we chose gradient descent, but other methods are applicable as well.

The most notable part in equation (1) are the mappings $P_{j}: V_{m} \rightarrow \mathbb{R}$, which perform the 1D-3D transformation step by simulating probe measurements at position/orientation $\left(p_{j}, o_{j}\right)$ from the moving image $T\left(V_{m}\right)$. It can also be thought of as a forward projection in tomography terms. This requires a model of the detection process in the probe device, modeling characteristics like detector sensitivity, collimation and shielding. In this work we employ a simple solid angle physical model of the detection process, modeling the field of view, angular detection as well as distance sensitivity analytically [4. Any source in $T\left(V_{m}\right)$ is modeled as a Poisson distribution dependent on the activity in $T\left(V_{m}\right)$, as is regularly done in emission tomography. In mathematical terms we discretize $T\left(V_{m}\right)=\left(x_{i}\right)_{i=1, \ldots, n}$ into $n$ regular isotropic voxels, then

$$
P_{j}\left(T\left(V_{m}\right)\right)=\sum_{i=1}^{n} \operatorname{model}\left(p_{j}, o_{j}, x_{i}\right),
$$

where $\operatorname{model}\left(p_{j}, o_{j}, x_{i}\right)$ denotes the physical model, that is the contribution of voxel $x_{i}$ to the measurement of the probe located at position/orientation $\left(p_{j}, o_{j}\right)$.

\section{Experiments and Results}

\subsection{Hardware Setup}

SPECT/CT imaging was provided by a Symbia T6 system (Siemens, Healthcare, Germany), intra-operative SPECT (in short: ioSPECT) by the declipseSPECT cart system (SurgicEye, Germany). The gamma detector employed was a Gamma-Probe System (Crystal Photonics, Germany), and tracking was performed using a Polaris Vicra infrared optical tracking system (Northern Digital, Canada) with reflective markers attached to both the gamma probe and to the scanning target (phantom or patient). 

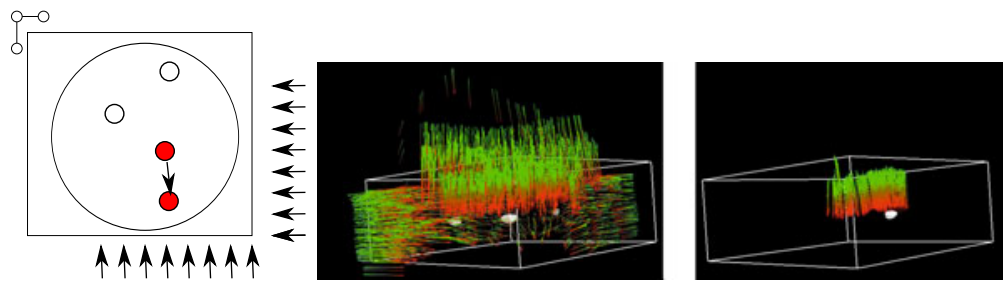

Fig. 2. Left: the phantom with three hollow spheres filled with a ${ }^{99 m} \mathrm{Tc}$ solution. The arrows illustrate scanning directions for ioSPECT, scanning from top not shown. The red sphere is moved by $2 \mathrm{~cm}$ to simulate deformation during surgery. Middle: full scan suited for reconstruction (arrows (green/red) indicating gamma probe measurements). Right: a fast search scan, with much less measurements and covering a much smaller region, used for registration.

\subsection{Phantom Experiments}

The phantom consists of a plastic cylinder mounted on a rectangular base plate, which also mounts the tracking target with reflective markers. Inside the cylinder three hollow spheres with a radius of $9.86 \mathrm{~mm}$ each are mounted at mid-height in a triangular fashion with a separation of $4 \mathrm{~cm}$ each. A Technetium- $99 \mathrm{~m}$ solution is filled into these spheres to simulate radioactive lymph nodes. The three spheres contained an activity of $1.6 \mathrm{MBq}, 1.6 \mathrm{MBq}$ and $1.65 \mathrm{MBq}$. No attenuating medium has been used. One of the spheres could be moved by $2 \mathrm{~cm}$ away from the other two spheres to simulate deformation during surgery, see Fig. 22 for an illustration.

Three series of experiments were performed on the phantom. Series 1: $V$ is a SPECT from the SPECT/CT device, 4 search scans (used as the 1D input $b_{j}$ for the registration) were performed. Series 2: $V$ is an ioSPECT with a search scan performed after reconstruction, repeated 4 times. Series 3: $V$ is an ioSPECT scan with a search scan performed after reconstruction, repeated 8 times. All the experiments were performed with the same equipment.

The SPECT $V$ was discretized into $128 \times 128 \times 55$ isotropic voxels of size $4.8 \mathrm{~mm}$. The registration of the SPECT to the tracking coordinate system was performed via the $\mathrm{CT}$ that was generated as part of the SPECT/CT acquisition (the SPECT and the CT are automatically co-registered). The ioSPECT used a $50 \times 50 \times 20$ volume for $V$ with isotropic voxels of size $2.5 \mathrm{~mm}$, the scanning protocol is illustrated in Fig. 2. The ioSPECT scans contained an average of 4832 measurements (min. 3576, max. 5445) with a scan duration between 4 and 5 minutes. The search scans all took about 30 seconds and contain 534 measurements on average (min. 513, max. 629). See also Fig. 2 for examples of a full scan versus a search scan. For all the experiments, the actual computation times of the registration process are on the order of seconds.

\subsection{Phantom Results}

We evaluate several types of errors. First, the accuracy of SPECT and ioSPECT compared to CT for series 1 and 2 is calculated using the euclidean distance 
Table 1. Results of the phantom experiments

\begin{tabular}{c||c|c|c|c} 
& $\begin{array}{c}\text { accuracy of } V \\
\text { compared to CT }\end{array}$ & ground truth & $\begin{array}{c}\text { 3D error } \\
\text { (average) }\end{array}$ & $\begin{array}{c}2 \mathrm{D} \text { in-plane error } \\
\text { (average) }\end{array}$ \\
\hline \hline series 1 (SPECT) & $4.6 \mathrm{~mm}$ & $\mathrm{CT}$ & $7.5 \pm 1.4 \mathrm{~mm}$ & $4.8 \pm 0.6 \mathrm{~mm}$ \\
series 2 (ioSPECT) & $11.9 \pm 6.6 \mathrm{~mm}$ & $\mathrm{CT}$ & $9.5 \pm 2.3 \mathrm{~mm}$ & $7.9 \pm 3.0 \mathrm{~mm}$ \\
series 2 (ioSPECT) & $11.9 \pm 6.6 \mathrm{~mm}$ & ioSPECT & $8.1 \pm 6.7 \mathrm{~mm}$ & $2.2 \pm 1.2 \mathrm{~mm}$ \\
series 3 (ioSPECT) & $\mathrm{n} / \mathrm{a}$ & ioSPECT & $7.1 \pm 2.8 \mathrm{~mm}$ & $4.2 \pm 2.9 \mathrm{~mm}$
\end{tabular}

between the centers of mass of the segmented spheres in both modalities. For all series, the 1D-3D registration was performed using the search scan of the moved phantom and compared the result to the ground truth. Here we computed two error measures, the positional euclidean error in $3 \mathrm{D}$, as well as a $2 \mathrm{D}$ euclidean error in a plane orthogonal to the surgeon's view. This latter error is more relevant in actual surgeries, as the surgeon is mainly interested in the location of nodes in the plane orthogonal to his viewing direction, since this determines the cutting direction, whereas a node that is located farther away but in the correct cutting direction is less of an issue. All the error types for series 1, 2 and 3 are summarized in Table 1.

For series 3, the search scan data was also tomographically reconstructed (using the same method as for ioSPECT) and then registered to the ioSPECT using a standard SSD-based 3D-3D registration. The 3D average error here was $30.4 \pm 13.8 \mathrm{~mm}$, the 2D average error was $15.2 \pm 7.0 \mathrm{~mm}$. This clearly shows that the search scan data is insufficient for tomographic treatment. In contrast, the error of the proposed 1D-3D registration method was $7.1 \pm 2.8 \mathrm{~mm}$ (3D average error) and $4.2 \pm 2.9 \mathrm{~mm}$ (2D in-plane average error), which is close to the inaccuracies of SPECT and ioSPECT themselves $(4.6 \mathrm{~mm}$ and $11.9 \pm 6.6 \mathrm{~mm}$ ). It is also interesting to note that the registration error using ioSPECT as ground truth is smaller than the one using CT as ground truth, as both ioSPECT and the search scan for registration use the same physical equipment with the same measurement error sources.

For series 3, we also studied the effect of using less than 500 average search scan data points, i.e. scanning with the probe for even less time. The measurements were thinned out, but still covered the same region, the results are outlined in Table 2. As long as the same area is covered, the registration error does not change significantly, even when just using $10 \%$ of the search scan data.

Finally for series 3 , a full ioSPECT data scan with an average of 4832 measurements was used as a "search scan", here the average 3D error was $6.3 \pm 2.5 \mathrm{~mm}$, the average $2 \mathrm{D}$ error was $3.7 \pm 2.4 \mathrm{~mm}$. This shows that when enough data is available to do a proper tomographic reconstruction, the results will be superior compared to our registration approach. However, in practical situations, having enough data for a reconstruction is not feasible due to the extra duration of the scanning and reconstruction procedure. 
Table 2. Results of phantom experiments of series 3 using less search scan data

\begin{tabular}{c|c||c|c}
$\begin{array}{c}\text { series 3 (ioSPECT) } \\
\text { (ground truth ioSPECT) }\end{array}$ & $\begin{array}{c}\text { no. of mea- } \\
\text { surements }\end{array}$ & $\begin{array}{c}\text { 3D error } \\
\text { (average) }\end{array}$ & $\begin{array}{c}2 \mathrm{D} \text { in-plane error } \\
\text { (average) }\end{array}$ \\
\hline \hline $100 \%$ search scan & 500 & $7.1 \pm 2.8 \mathrm{~mm}$ & $4.2 \pm 2.9 \mathrm{~mm}$ \\
$50 \%$ search scan & 250 & $7.0 \pm 2.7 \mathrm{~mm}$ & $4.2 \pm 2.7 \mathrm{~mm}$ \\
$33 \%$ search scan & 165 & $6.9 \pm 2.9 \mathrm{~mm}$ & $4.2 \pm 3.1 \mathrm{~mm}$ \\
$25 \%$ search scan & 125 & $6.7 \pm 2.6 \mathrm{~mm}$ & $4.0 \pm 2.7 \mathrm{~mm}$ \\
$20 \%$ search scan & 100 & $6.8 \pm 2.5 \mathrm{~mm}$ & $4.6 \pm 2.8 \mathrm{~mm}$ \\
$10 \%$ search scan & 50 & $6.5 \pm 2.7 \mathrm{~mm}$ & $4.5 \pm 2.7 \mathrm{~mm}$
\end{tabular}

\subsection{Experiment with Patient Data}

For a more realistic evaluation, we test the registration method on patient data as well. For this an ioSPECT of a breast cancer patient undergoing sentinel lymph node biopsy was used with 7078 measurements reconstructed into a $40 \times 50 \times 25$ volume with isotropic voxels of size $5 \mathrm{~mm}$ (see Fig. 1). The radioactive lymph node was segmented and artificially moved $40 \mathrm{~mm}$ from its original position, similar to the phantom experiment. We perform a search scan of 350 measurements on the patient to register the artificially moved nodes back to their original position. The resulting 3D error with respect to the ioSPECT ground truth is $4.2 \mathrm{~mm}$ and the $2 \mathrm{D}$ error is $4.2 \mathrm{~mm}$ as well.

\section{Discussion}

The accuracy of the proposed registration method is clinically acceptable using ioSPECT as ground truth, since the accuracy of clinical SPECT is between $5 \mathrm{~mm}$ to $12 \mathrm{~mm}$. As one of the aims is to update ioSPECT data, using it as ground truth seems most appropriate. When using pre-operative CT as the ground truth, results suffer, as tracking errors have a higher impact in this scenario. Our evaluation on the patient data set is satisfactory. Even though gathering intra-operative patient data is complex, we plan to investigate further into this direction, in particular analyzing shorter and shorter scan times.

The registration accuracy is limited by the inherent resolution limitations of functional imaging as well as the tracking inaccuracies. However the quality of the input volume used as prior information is also crucial. Intra-operative SPECT scans vary in quality, especially if performed by inexperienced operators. Please note that the flexibility offered by the intra-operative SPECT comes at such a cost. Such compromises within surgical environments are acceptable since the information is provided not to a surgical robotic system, but to an expert surgeon in order to improve his or her performance.

\section{Conclusion}

To our knowledge this is the first work proposing a $1 \mathrm{D}-3 \mathrm{D}$ registration method for intra-operative functional imaging, which allows registering $3 \mathrm{D}$ volumes to 
1D measurements with an accuracy suitable for a surgical setting. Our approach provides 3-dimensional image data but requires less readings, and is therefore faster, than a complete scan. Together with upcoming developments of intelligent surgical instruments including integrated detectors this method will enable realtime updates for the guidance of surgeons towards deep-seated targets, resulting in faster and more efficient surgeries and a better quality control.

Acknowledgements. The authors would like to thank Alexander Hartl and Asli Okur for their help in performing the experiments. This work was partially funded by the SFB 824 of the Deutsche Forschungsgesellschaft (DFG), the Graduate School of Information Science in Health (GSISH) and the TUM Graduate School.

\section{References}

1. Baumhauer, M., Feuerstein, M., Meinzer, H.P., Rassweiler, J.: Navigation in endoscopic soft tissue surgery: perspectives and limitations. J. Endourol. 22(4), 751-766 (2008)

2. Buck, A.K., Nekolla, S., Ziegler, S., Beer, A., Krause, B.J., Herrmann, K., Scheidhauer, K., Wester, H.J., Rummeny, E.J., Schwaiger, M., Drzezga, A.: SPECT/CT. J. Nucl. Med. 49(8), 1305-1319 (2008)

3. Czernin, J., Benz, M.R., Allen-Auerbach, M.S.: PET/CT imaging: The incremental value of assessing the glucose metabolic phenotype and the structure of cancers in a single examination. Eur. J. Radiol. 73(3), 470-480 (2010)

4. Hartl, A., Ziegler, S.I., Navab, N.: Models of detection physics for nuclear probes in freehand spect reconstruction. In: IEEE Medical Imaging Conference, Knoxville, USA (October 2010)

5. Lyman, G., Giuliano, A., Somerfield, M., Benson III, A.B., Bodurka, D.C., Burstein, H.J., Cochran, A.J., Cody III, H.S., Edge, S.B., Galper, S., Hayman, J.A., Kim, T.Y., Perkins, C.L., Podoloff, D.A., Sivasubramaniam, V.H., Turner, R.R., Wahl, R., Weaver, D.L., Wolff, A.C., Winer, E.P.: American society of clinical oncology guideline recommendations for sentinel lymph node biopsy in early-stage breast cancer. J. Clin. Oncol. 23, 7703-7720 (2005)

6. Valdes Olmos, R.A., Vidal-Sicart, S., Nieweg, O.E.: Technological innovation in the sentinel node procedure: towards 3 -d intraoperative imaging. Eur. J. Nucl. Med. Mol. Imaging 37(8), 1449-1451 (2010)

7. Wendler, T., Herrmann, K., Schnelzer, A., Lasser, T., Traub, J., Kutter, O., Ehlerding, A., Scheidhauer, K., Schuster, T., Kiechle, M., Schwaiger, M., Navab, N., Ziegler, S.I., Buck, A.K.: First demonstration of 3-d lymphatic mapping in breast cancer using freehand spect. Eur. J. Nucl. Med. Mol. Imaging (March 2010) 